\title{
A trace bound for integer-diagonal positive semidefinite matrices
}

https://doi.org/10.1515/spma-2020-0002

Received September 27, 2019; accepted December 2, 2019

Abstract: We prove that an $n$-by- $n$ complex positive semidefinite matrix of rank $r$ whose graph is connected, whose diagonal entries are integers, and whose non-zero off-diagonal entries have modulus at least one, has trace at least $n+r-1$.

Keywords: positive semidefinite matrices, integer-diagonal, trace

MSC: 15B48, 15A15, 05C50

\section{Introduction}

The graph of an $n$-by- $n$ Hermitian matrix $M=\left(m_{i j}\right)$ has vertex set $\{1,2, \ldots, n\}$ and edge set $\left\{i j \mid i<j, m_{i j} \neq\right.$ $0\}$. As part of their work on the Schur-Siegel-Smyth problem for totally positive algebraic integers, James McKee and Pavlo Yatsyna [3] proved that an $n$-by- $n$ positive definite matrix $S$ whose entries are integers and whose graph is connected must have trace at least $2 n-1$. As a consequence, 2 is the smallest limit point of the absolute trace (which for an $n$-by- $n$ matrix is the trace divided by $n$ ) of such matrices.

The integer entries are important to McKee and Yatsyna's proof: since $S$ is positive definite, it can be factored as $S=B^{\mathrm{T}} B$, and thus viewed as the Gram matrix of the columns $x_{1}, x_{2}, \ldots, x_{n}$ of $B$. In a minimaltrace connected counterexample, we can assume without loss of generality that $x_{1}$ is a unit vector. Then the Gram matrix of $x_{1}, x_{2}^{\prime}, x_{3}, \ldots, x_{n}$, where $x_{2}^{\prime}=x_{2}-\left(x_{1}^{\mathrm{T}} x_{2}\right) x_{1}$, still has integer entries and eventually provides a contradiction.

Are the integer entries necessary? In this note, we prove a generalization for complex positive semidefinite matrices and show that while the diagonal entries must be integers, the off-diagonal non-zero entries need only have modulus at least 1. A generalization of McKee and Yatsyna's absolute trace result follows as a corollary.

In addition to standard tools and definitions from matrix analysis [2] and graph theory [1], one fact we will use repeatedly is that, because the sum of a positive definite matrix and a positive semidefinite matrix is still positive definite, adding a positive number to a diagonal entry of a positive definite matrix results in another positive definite matrix. Also note that an empty graph on a single vertex is connected.

\section{New Results}

Lemma 1. An $n$-by- $n$ complex positive definite matrix whose graph is a tree, whose diagonal entries are integers, and whose non-zero off-diagonal entries have modulus at least one, has trace at least $2 n-1$.

^Corresponding Author: Lon Mitchell: Lon Mitchell, University of South Florida St. Petersburg, Florida, USA, E-mail: lonmitchell@usfsp.edu 
Proof. Proceed by induction on $n$, noting the result is true for $n=1$. Assume that the result is true for all $k$-by$k$ matrices where $1 \leq k<n$, and let $M=\left(m_{i j}\right)$ be an $n$-by- $n$ positive definite matrix whose graph is a tree with vertices labeled $v_{1}, v_{2}, \ldots, v_{n}$ corresponding to the rows of $M$. Assume for the sake of eventual contradiction that the trace of $M, \operatorname{tr} M$, is less than $2 n-1$.

Since the graph $G$ of $M$ is a tree, it has a pendant vertex (a vertex of degree one). Without loss of generality, we can assume vertex $v_{1}$ has unique neighbor $v_{2}$. If the diagonal element $m_{11}$ of $M$ is greater than 1 , then applying the induction hypothesis to $M_{11}$, the matrix obtained from $M$ by deleting the first row and column, yields $\operatorname{tr} M \geq 2+\operatorname{tr} M_{11} \geq 2+2(n-1)-1=2 n-1$, a contradiction. So we may assume $m_{11}=1$. Since $v_{1}$ is pendant,

$$
M=\left[\begin{array}{cc}
1 & \bar{\alpha} e_{1}^{*} \\
\alpha e_{1} & M_{11}
\end{array}\right],
$$

where $\alpha$ is a complex number with $|\alpha| \geq 1$ and $e_{1}$ is the standard basis vector.

Consider next the Schur complement $M^{\prime}=M_{11}-|\alpha|^{2} e_{1} e_{1}^{\mathrm{T}}$, which is an $(n-1)$-by- $(n-1)$ positive definite matrix. All off-diagonal elements of $M^{\prime}$ remain unchanged from the corresponding entries of $M$, so the graph of $M^{\prime}$ is a tree. All main-diagonal elements of $M^{\prime}$ also remain unchanged with the exception of $m_{11}^{\prime}=m_{22}$ $|\alpha|^{2} \leq m_{22}-1$.

Since $m_{11}^{\prime}$ may not be an integer, let $M^{\prime \prime}$ be the matrix obtained from $M^{\prime}$ by replacing $m_{11}^{\prime}$ with $m_{11}^{\prime \prime}=$ $m_{22}-1$. Since $m_{11}^{\prime \prime} \geq m_{11}^{\prime}, M^{\prime \prime}$ is also positive definite. Further, its graph is a tree, its diagonal entries are integers, and its non-zero off-diagonal entries have modulus at least one. Finally, $\operatorname{tr} M^{\prime \prime}=\operatorname{tr} M-2<2 n-3$, a contradiction of the induction hypothesis. Thus $\operatorname{tr} M \geq 2 n-1$.

Theorem 1. An $n$-by- $n$ complex positive definite matrix whose graph is connected, whose diagonal entries are integers, and whose non-zero off-diagonal entries have modulus at least one, has trace at least $2 n-1$.

Proof. Proceed by induction on $n$, noting the result is true for $n=1$. Assume that the result is true for all $k$-by- $k$ matrices where $1 \leq k \leq n-1$, and let $M$ be an $n$-by- $n$ positive definite matrix whose diagonal entries are integers, whose graph is connected, and whose non-zero off-diagonal entries have modulus at least one.

Assume for the sake of eventual contradiction that $\operatorname{tr} M<2 n-1$. By adding to a diagonal entry if needed, we can assume that we have a matrix $M$ with the above-mentioned properties and with $\operatorname{tr} M=2 n-2$.

Let $G$ be the graph of $M$ and let $m_{v}$ be the diagonal entry of $M$ corresponding to vertex $v$ in $G$. For each vertex $v$ of $G$, let $c(v)$ be the number of connected components of $G \backslash v$.

Suppose first that there is a vertex $v$ of $G$ such that $m_{v}>c(v)$. Consider $M(v)$, the matrix obtained from $M$ by removing the row and column corresponding to $v$. Applying the induction hypothesis to the principal submatrices $M_{1}, M_{2}, \ldots, M_{c(v)}$ of $M(v)$ corresponding to the connected components $C_{1}, C_{2}, \ldots, C_{c(v)}$ of $G \backslash v$, we find that

$$
\begin{aligned}
\operatorname{tr} M=m_{v}+\operatorname{tr} M(v) & =m_{v}+\sum_{i=1}^{c(v)} \operatorname{tr} M_{i} \\
& \geq m_{v}+\sum_{i=1}^{c(v)}\left(2\left|C_{i}\right|-1\right)=m_{v}-c(v)+2(n-1) \geq 2 n-1 .
\end{aligned}
$$

Thus we must have that $m_{v} \leq c(v)$ for each vertex $v$.

Let $T$ be a spanning tree of $G$. Since $T$ is a tree on $n$ vertices, it has $n-1$ edges, and so

$$
\sum_{v \in G} d_{T}(v)=2(n-1)
$$

where $d_{T}(v)$ is the degree of the vertex $v$ in $T$. Since $d_{T}(v) \geq c(v) \geq m_{v}$ for each $v$ but

$$
\sum_{v \in G} d_{T}(v)=2(n-1)=\operatorname{tr} M=\sum_{v \in G} m_{v}
$$

we must have $d_{T}(v)=c(v)=m_{v}$ for each $v$. 
For any vertex $v$ of $G$, because $d_{T}(v)=c(v)$, there is a bijective correspondence between the neighbors of $v$ in $T$ and the connected components of $G \backslash v$. Thus, if vertices $v_{i}$ and $v_{j}$ are not adjacent in $T$, then they belong to different connected components of $G \backslash w$ for any vertex $w$ on a path between them in $T$, and so are not adjacent in $G$ either. So, in fact, $G=T$, and Lemma 1 requires $\operatorname{tr} M \geq 2 n-1$, contradicting our earlier assumption. Thus $\operatorname{tr} M \geq 2 n-1$.

Corollary 1. The smallest limit point of the set of absolute traces of matrices satisfying the conditions of Theorem 1 is 2 .

Remark. The matrices $\left[\begin{array}{ll}1 & 0 \\ 0 & 1\end{array}\right],\left[\begin{array}{ll}1.0 & 0.5 \\ 0.5 & 1.0\end{array}\right]$, and $\left[\begin{array}{ll}1.1 & 1.0 \\ 1.0 & 1.1\end{array}\right]$ show that none of the conditions of Theorem 1 can be removed.

Theorem 2. An $n$-by- $n$ complex positive semidefinite matrix of rank $r$ whose graph is connected, whose diagonal entries are integers, and whose non-zero off-diagonal entries have modulus at least one, has trace at least $n+r-1$.

Proof. Proceed by induction on the nullity. The nullity zero case is Theorem 1 . Assume the result is true for all nullities less than some $k>0$. Let $M$ be an $n$-by- $n$ complex positive semidefinite matrix of nullity $k=n-r$ whose graph is connected, whose diagonal entries are integers, and whose non-zero off-diagonal entries have modulus at least one.

Consider $M$ as the Gram matrix of linearly dependent vectors $x_{1}, x_{2}, \ldots, x_{n}$ in $\mathbb{C}^{n}$. Let $l$ be such that $x_{l}$ is in the span of the other vectors, and let $y$ be a unit vector in $\mathbb{C}^{n}$ orthogonal to each $x_{i}$. Then the Gram matrix $M^{\prime}$ of $x_{1}, x_{2}, \ldots, x_{l-1}, x_{l}+y, x_{l+1}, \ldots, x_{n}$ is equal to $M$ except for an increase of 1 in the $m_{l l}$ main-diagonal element, so its graph is connected, its diagonal entries are integers, and the non-zero off-diagonal entries have modulus at least one. The nullity of $M^{\prime}$ is $k-1$, so by the induction hypothesis and by construction, $\operatorname{tr} M=\operatorname{tr} M^{\prime}-1 \geq(n+(r+1)-1)-1=n+r-1$.

Corollary 2. An $n$-by- $n$ complex positive semidefinite matrix of rank $r$ whose graph has $s$ connected components, whose diagonal entries are integers, and whose non-zero off-diagonal entries have modulus at least one, has trace at least $n+r-s$.

Acknowledgement: Publication of this article was funded by the University of South Florida St. Petersburg's Open Access Publication Fund.

\section{References}

[1] Reinhard Diestel, Graph theory, 5th ed., Graduate Texts in Mathematics, 173, Springer, Berlin, 2018.

[2] Roger A. Horn, Charles R. Johnson, Matrix analysis, 2nd ed., Cambridge University Press, Cambridge, 2013.

[3] James McKee, Pavlo Yatsyna, A trace bound for positive definite connected integer symmetric matrices, Linear Algebra Appl., 444, 227-230, 2014. 